\title{
Selective lymphadenectomy of para-aortic lymph nodes for advanced gastric cancer
}

\author{
TAKASHI FUJIMURA, KEISHI NAKAMURA, KATSUNOBU OYAMA, HIROSHI FUNAKI, \\ HIDETO FUJITA, SHINICHI KINAMI, ITASU NINOMIYA, SACHIO FUSHIDA, \\ GENICHI NISHIMURA, MASATO KAYAHARA and TETSUO OHTA
}

Gastroenterologic Surgery, Kanazawa University Hospital, Kanazawa, Ishikawa 920-8641, Japan

Received February 3, 2009; Accepted March 13, 2009

DOI: $10.3892 /$ or_00000464

\begin{abstract}
The Japanese randomized trial comparing standard D2 with D2 plus additional para-aortic lymph node (PAN) dissection for advanced gastric cancer (JCOG study 9501) did not demonstrate any difference in survival between the two groups. It is unknown whether there is any prognostic benefit in dissection for subgroups of PAN. Non-inferiority in survival of the patients with PAN metastasis to the patients having $\mathrm{n} 2$ metastasis was examined according to the subgroup of PANs and the tumor location. The survival curve of $n 2$ patients $(n=131)$ were retrospectively compared with that of patients with PAN metastasis $(n=55)$ and also compared with that of patients with metastasis to subgroup of PANs by the location of primary tumor (regions U, M and L). Expectedly, the prognosis of the $\mathrm{n} 2$ patients is significantly better than that of the patients with PAN metastasis, but there was no difference in the survival times between the $\mathrm{n} 2(+)$ group and the a2-lat $(+)$ or the b1-int $(+)$ group, suggesting that the a2-lat or the b1-int dissection matched the D2 dissection. Furthermore, the importance in dissection of the a2-lat and the b1-int was investigated according to the primary tumor location. The patients with metastasis to a2-lat in the region $\mathrm{U}$, a2-lat and b1-int in the region $\mathrm{M}$ and b1-int in the region $\mathrm{L}$ demonstrated prognostic non-inferiority to the patients having $\mathrm{n} 2$ metastasis. Selective lymphadenectomy of subgroups of PANs in which metastases are highly suspected according to the tumor location is one of treatment strategies to advanced gastric cancer.
\end{abstract}

\section{Introduction}

Though the incidence of gastric cancer has decreased in the world, it is still one of leading causes of cancer death in many

Correspondence to: Dr Takashi Fujimura, Gastroenterologic Surgery, Kanazawa University Hospital, 13-1 Takaramachi, Kanazawa, Ishikawa 920-8641, Japan

E-mail: tphuji@surg2.m.kanazawa-u.ac.jp

Key words: para-aortic lymph node, extended lymphadenectomy, gastric cancer countries of different areas, such as Eastern Asia, Eastern Europe and Latin America. Hematological and peritoneal metastases in gastric cancer are life-threatening diseases, which should be treated with chemotherapy. It is probable that surgical intervention is able to improve prognosis of the patients with lymph node metastasis. Various types of lymph node dissection including D1, D2 and D3 have been tried to control lymph node metastasis in gastric cancer. Extended lymphadenectomy disappointed the expectation of survival improvement by two European randomized control studies comparing the D1 procedure with the D2 procedure $(1,2)$, whereas another randomized trial reported in 2006 showed a significant benefit in patient survival for a D2 or D3 procedure as compared with D1 dissection, without increased operative mortality (3). It was reported that $20-30 \%$ of patients with advanced gastric cancer had metastasis to paraaortic lymph nodes (PANs) (4) and the 5-year survival of such patients reached 13 to $40 \%$ (5-7). These data facilitated to launch a randomized study on the importance of dissection of PANs. The Japanese randomized trial comparing standard D2 with D2 plus additional para-aortic lymphadenectomy for advanced gastric cancer (JCOG study 9501) was carried out between July 1995 and April 2001. Unfortunately, the JCOG study 9501 did not demonstrate any difference in survival between the two groups (8).

It remains unknown whether there is any prognostic benefit in subsets into which patients are classified according to primary tumor location or subgroup of PAN. Non-inferiority in survival of the patients with PAN metastasis to the patients having metastasis in the second-tier lymph nodes (n2) was retrospectively examined according to the subgroup of PANs and the tumor location.

\section{Patients and methods}

All patients enrolled in this study were histologically proven gastric adenocarcinoma. A total of 937 patients who were assessed equal to or higher than clinical stage II received D2 $(n=715)$ or D3 $(n=222)$ surgery with the grade of residual tumor, R0 and R1 (9), in Kanazawa University Hospital between April, 1973 and December, 2002. D3 operation was defined as D2 plus PAN lymphadenectomy in the present study. PAN was subgrouped into a1, a2-lat, a2-int, b1-lat, b1-int and b2 (Fig. 1). The clinical and pathological findings except for factors $\mathrm{H}$ and $\mathrm{P}$ were based on the guidelines of the 


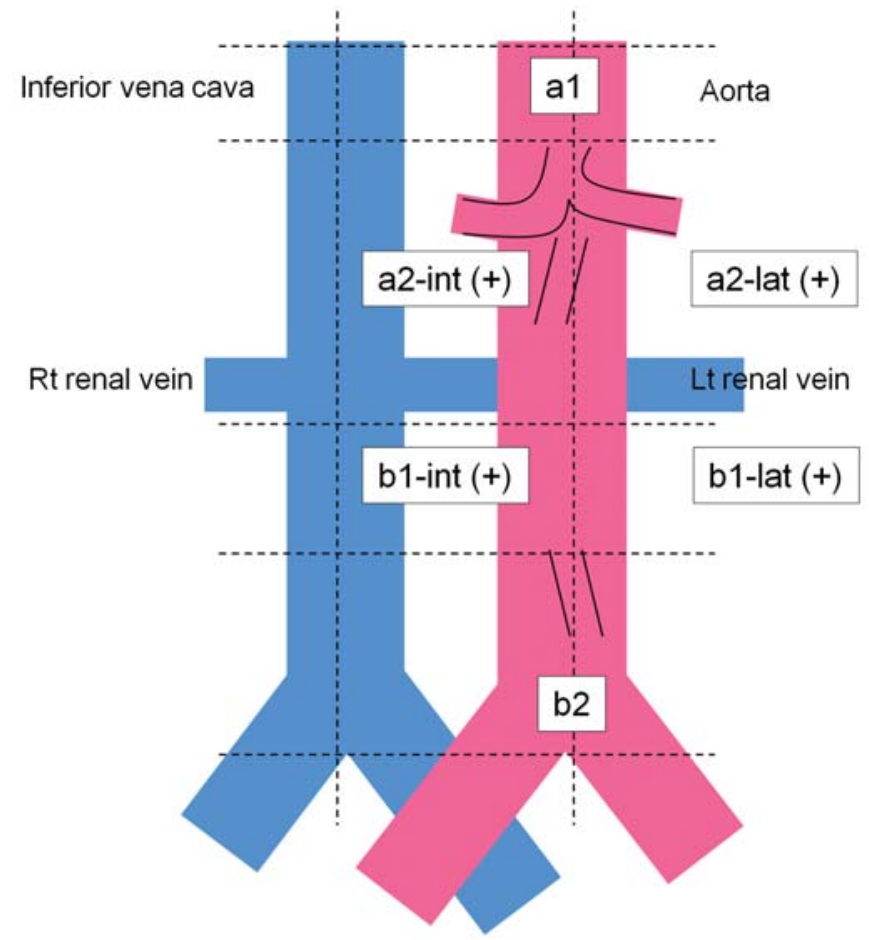

Figure 1. Subgroup of the para-aortic lymph nodes. The 'a1' lymph node is defined as node located between aortic foramen and upper margin of celiac axis in the craniocaudal direction. The 'a2' lymph node is defined as node located between upper margin of celiac axis and lower margin of left renal vein (LRV). The 'b1' lymph node is defined as node located between lower margin of LRV and upper margin of inferior mesenteric artery (IMA). The ' $b 2$ ' lymph node is defined as node located between upper margin of IMA and bifurcation of abdominal aorta. The '-int' and '-lat' lymph nodes in the lateral direction are defined as nodes located between center of aorta and center of inferior vena cava and nodes located on the left side to center of aorta, respectively.

second edition of General Rules of Gastric Cancer, edited by the Japanese Research Society for Gastric Cancer (10). The factors $\mathrm{H}$ and $\mathrm{P}$ were described according to the guidelines of the first edition (11).

To examine metastatic rates in subgroups of the PANs, 222 patients receiving D3 operation were analyzed. Survival curve of patients with PAN metastasis [PAN (+) patients] was compared with that of patients with metastasis to $n 2$ [n2 (+) patients]. A total of $131 \mathrm{n} 2(+)$ patients consisted of 71 and 60 patients undergoing D2 and D3 operations, respectively, while all 55 PAN (+) patients received D3 operation. Furthermore, survival curves of $n 2(+)$ patients and patients with metastasis to subgroup of PANs were compared by the location of primary tumor (regions $\mathrm{U}, \mathrm{M}$ and $\mathrm{L}$ ).

The significant difference in proportions between groups was determined with the Chi-square test. Patient survival was calculated with the Kaplan-Meier method and survival curves were compared with the log-rank method. Statistical significance was defined as a p-value $<0.05$.

\section{Results}

Patient demographics of $\mathrm{n} 2(+)$ patients and PAN (+) patients is summarized in Table I. The patients in the n 3 group had tumor with deep invasion, peritoneal dissemination, extended level of lymph node metastasis intraoperatively estimated ( $\mathrm{sN}$ )
Table I. Patient characteristics.

\begin{tabular}{|c|c|c|c|}
\hline & $\begin{array}{c}\mathrm{n} 2(+) \\
(\mathrm{n}=131)\end{array}$ & $\begin{array}{c}\operatorname{PAN}^{\mathrm{a}}(+) \\
\quad(\mathrm{n}=55)\end{array}$ & P-value \\
\hline \multicolumn{4}{|l|}{ Gender } \\
\hline Man/Woman & $79 / 52$ & $34 / 21$ & 0.98 \\
\hline Average age (range) & $59(19-70)$ & $59(18-71)$ & 0.42 \\
\hline \multicolumn{4}{|l|}{ Gross type } \\
\hline 0 & 11 & 3 & 0.091 \\
\hline 1 & 5 & 1 & \\
\hline 2 & 48 & 12 & \\
\hline 3 & 42 & 28 & \\
\hline 4 & 14 & 9 & \\
\hline 5 & 11 & 2 & \\
\hline \multicolumn{4}{|l|}{ Tumor location } \\
\hline $\mathrm{U} / \mathrm{M} / \mathrm{L}$ & $37 / 44 / 50$ & $19 / 16 / 20$ & 0.67 \\
\hline \multicolumn{4}{|l|}{ Tumor depth } \\
\hline $1 / 2 / 3 / 4$ & $33 / 19 / 58 / 21$ & $4 / 6 / 30 / 15$ & 0.018 \\
\hline \multicolumn{4}{|l|}{$\mathrm{H}$} \\
\hline $0 / 1$ & $130 / 1$ & $53 / 2$ & 0.43 \\
\hline \multicolumn{4}{|l|}{$\mathrm{P}$} \\
\hline $0 / 1$ & $123 / 8$ & $45 / 10$ & 0.023 \\
\hline \multicolumn{4}{|l|}{$\mathrm{sN}$} \\
\hline $0 / 1 / 2 / \mathrm{PAN}^{\mathrm{a}}$ & $14 / 37 / 68 / 12$ & $0 / 13 / 15 / 27$ & $<0.001$ \\
\hline \multicolumn{4}{|l|}{ Histology } \\
\hline $\mathrm{D} / \mathrm{Ud} / \mathrm{Sq}^{\mathrm{b}}$ & $65 / 65 / 1$ & $16 / 39 / 0$ & 0.026 \\
\hline \multicolumn{4}{|l|}{ Surgery } \\
\hline Total gastrectomy & 61 & 41 & $<0.001$ \\
\hline Distal gastrectomy & 60 & 8 & \\
\hline Proximal gastrectomy & 6 & 1 & \\
\hline Pancreatoduodenectomy & 3 & 3 & \\
\hline Others & 1 & 2 & \\
\hline
\end{tabular}

PAN $^{\mathrm{a}}$, Para-aortic lymph node; $\mathrm{D} / \mathrm{Ud} / \mathrm{Sq}^{\mathrm{b}}$, differentiated/undifferentiated/squamous.

and undifferentiated-type cancer, comparing with those in the $\mathrm{n} 2$ group. Distal gastrectomy in the $\mathrm{n} 2(+)$ group was more frequently performed than in the PAN $(+)$ group.

Metastatic rates in subgroups of the PANs were as follows; 9.4\% (20/212) in a2-lat, 6.5\% (13/199) in a2-int, 7.4\% (14/188) in b1-lat, 6.5\% (13/200) in b1-int, 7.7\% (1/13) in a1 and 57\% $(4 / 7)$ in b2. In the D2 group, 472 patients showed n0; 114, n1; $107, \mathrm{n} 2$ and $20, \mathrm{n} 3$, while 51 patients showed n0; 56, n1; 60, $\mathrm{n} 2$ and $55, \mathrm{n} 3$, in the D3 group. The metastasis to PANs in the D2 group was diagnosed by sampling of PANs.

The survival was compared between the PAN (+) patients and the $\mathrm{n} 2(+)$ patients (Fig. 2). The 1- and 5-year survival rates of the $\mathrm{n} 2(+)$ patients were 78 and $43 \%$, while the 1 - and 5 -year survival rates of the PAN $(+)$ patients were 63 and $22 \%$. The prognosis of the $\mathrm{n} 2(+)$ patients is significantly better than that of the PAN (+) patients. Then, the survival curve of the $\mathrm{n} 2(+)$ patients was compared with that of the patients with metastasis to each subgroup of PANs (Fig. 3). The 1- and 5-year survival rates of the patients with metastasis 


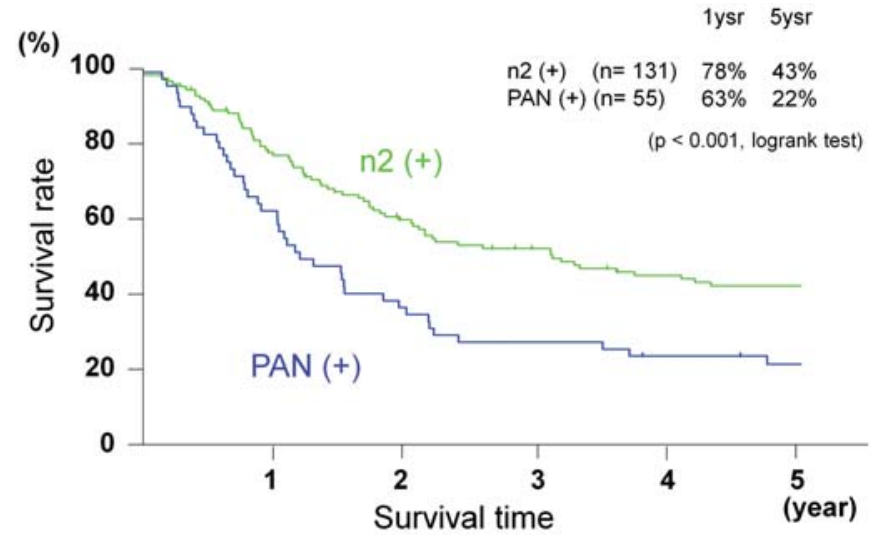

Figure 2. Survival curves of the n2 (+) group and the PAN (+) group.

in a 2 -lat or b1-int were 60 and $35 \%$ or 92 and $54 \%$, respectively. There was no difference in the median survival times between the $\mathrm{n} 2(+)$ group and the a2-lat $(+)$ or the b1int (+) group, suggesting that the a2-lat or the b1-int dissection matched the D2 dissection in the prognosis. On the other hand, the 1- and 5-year survival rates of the patients with metastasis in a2-int or b1-lat were 62 and $15 \%$ or 50 and $14 \%$, respectively, thus significantly lower than those of the patients with the $\mathrm{n} 2(+)$ group. These data indicated that the a2-lat and the b1-int were candidates for selective lymphadenectomy in PAN dissection.

Furthermore the importance in dissection of the a2-lat and the b1-int was investigated according to the primary tumor location. The 1- and 5-year survival rates of the $\mathrm{n} 2(+)$ patients were 77 and $36 \%$, respectively, while these rates of the patients with metastasis in a2-lat were 86 and $57 \%$, respectively, in region $U$ (Fig. 4). There was no difference in the median survival times between the $n 2(+)$ group and the a2-lat (+) group, suggesting that the a2-lat dissection matched the D2 dissection in the prognosis in region $\mathrm{U}$. Though the survival time of the b1-int (+) group was marginally shorter than that of the $\mathrm{n} 2(+)$ group, no conclusion could be made because of very few patients with the b1-int (+) group. Similar analyses were carried out for the cancers of regions $\mathrm{M}$ and $\mathrm{L}$ (Figs. 5 and 6). These analyses indicated that the a2-lat and b1-int dissection in the region $\mathrm{M}$ and the b1-int dissection in region $\mathrm{L}$ matched the $\mathrm{D} 2$ dissection in the prognosis.

\section{Discussion}

The Japanese randomized trial for D2 plus para-aortic lymphadenectomy in advanced gastric cancer (JCOG study 9501) failed in showing prognostic benefit. However, only $8.5 \%$ of patients had pathological metastasis in PANs and the 5-year survival rate reached $18.2 \%$ (8). The positive rate of PAN-metastasis in the JCOG study was much lower than expected, while the survival rate of their study is consistent with ones of previous studies. The 5-year survival rate of patients with liver metastases or peritoneal dissemination is as low as $<5 \%$, much worse than that of patients with PAN metastasis. These results supported that dissection of PANs is likely to improve the survival of the patients with PAN metastasis other than liver metastases or peritoneal dissemination. Taken together, the JCOG study 9501 could not prove superiority in the prognosis of patients with PANmetastasis receiving PAN dissection to D2 dissection, but only
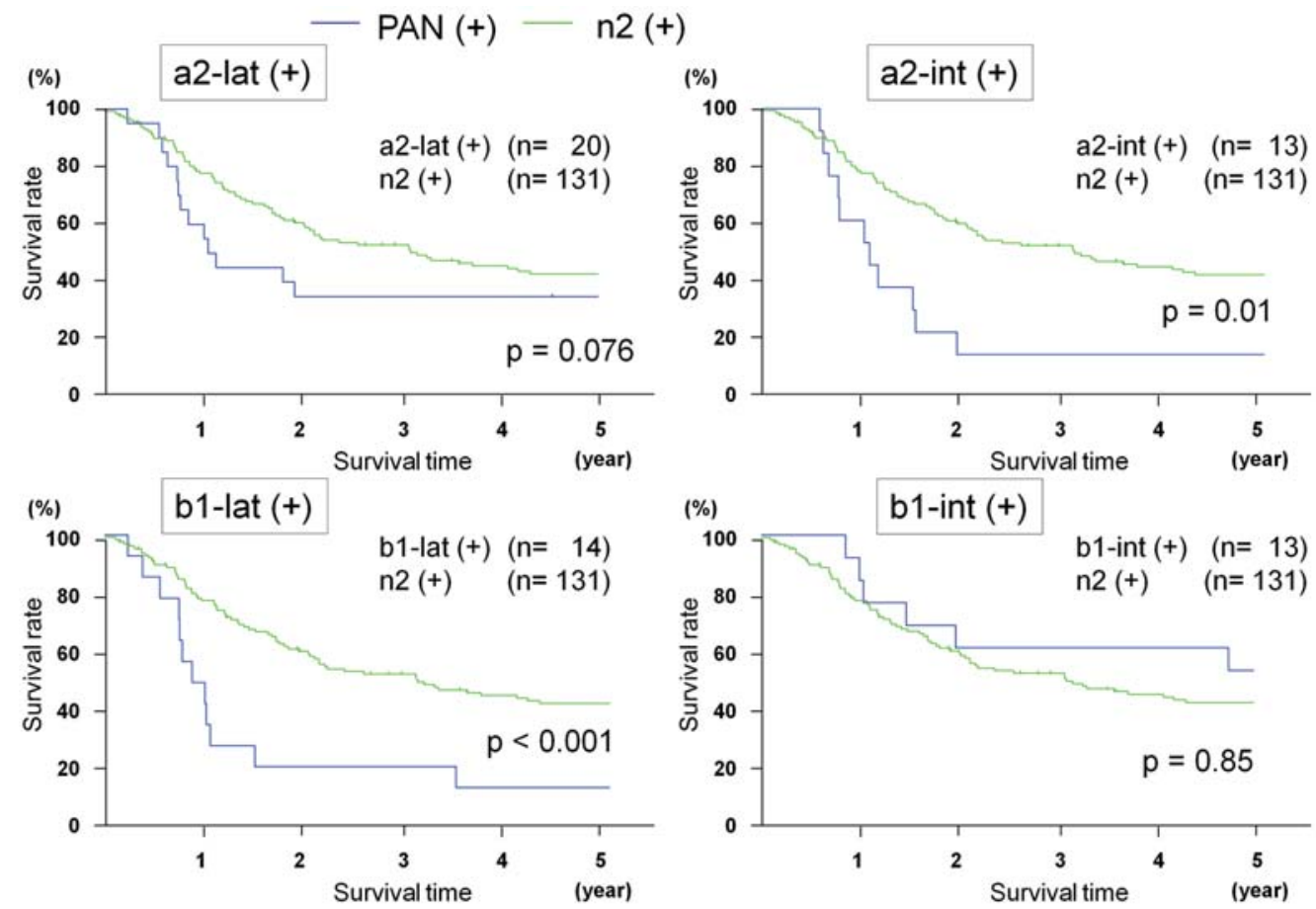

Figure 3. Survival curves of the $\mathrm{n} 2(+)$ group and the PAN (+) group according to the subgroup of PANs. There was no difference in the median survival times between the $\mathrm{n} 2(+)$ group and the a2-lat $(+)$ or the b1-int $(+)$ group, while the survival time of the n2 (+) group was significantly longer than that of the a2-int $(+)$ or b1-lat (+) group. 


\section{Upper-third (the region U)}

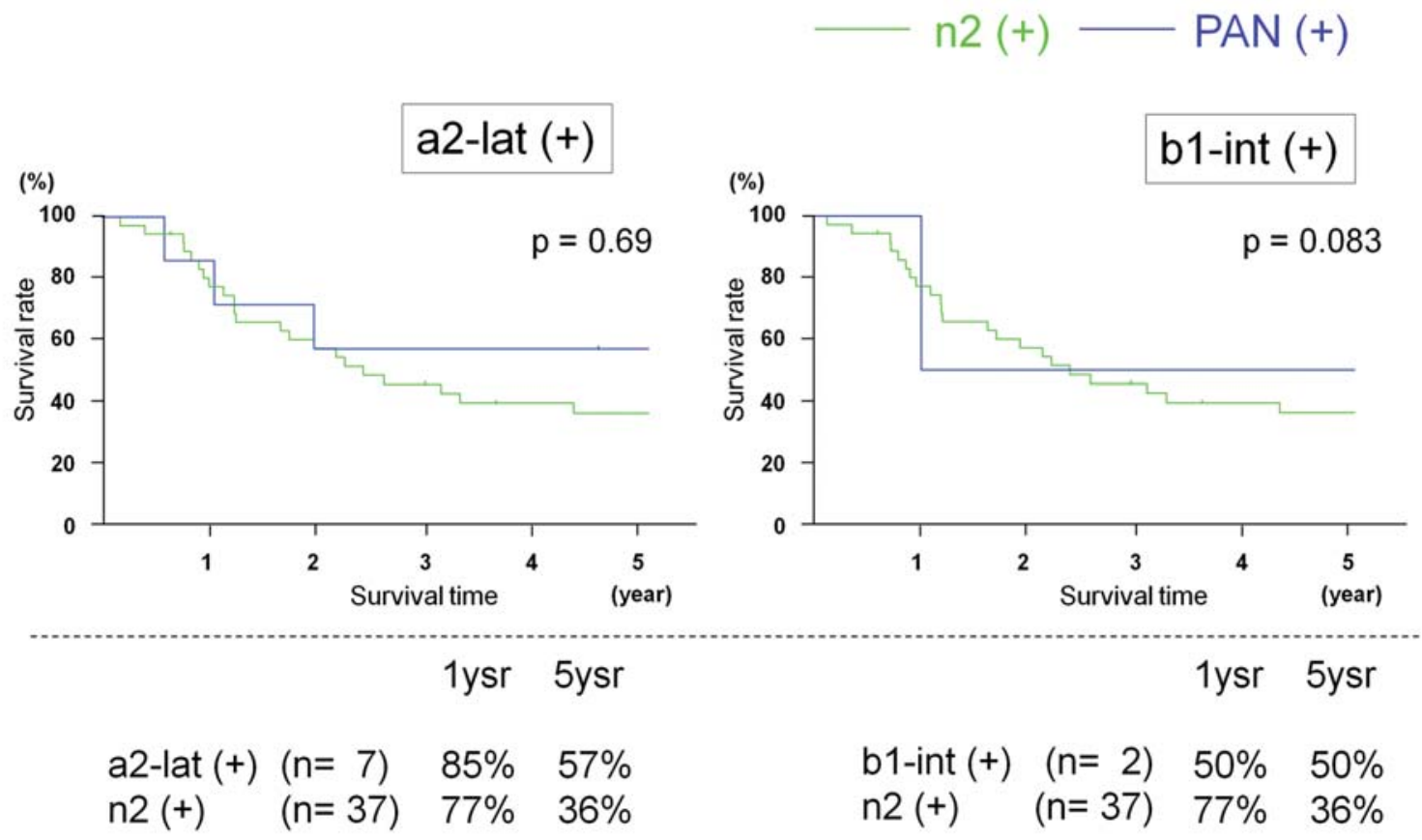

Figure 4. Survival curves of the n2 (+) group and the a2-lat $(+)$ or the b1-int $(+)$ group of the upper-third gastric cancer. There was no difference in the median survival times between the $\mathrm{n} 2(+)$ group and the a2-lat $(+)$ group. The survival time of the b1-int $(+)$ group was marginally shorter than that of the $2(+)$ group.

\section{Middle-third (the region $\mathrm{M}$ )}
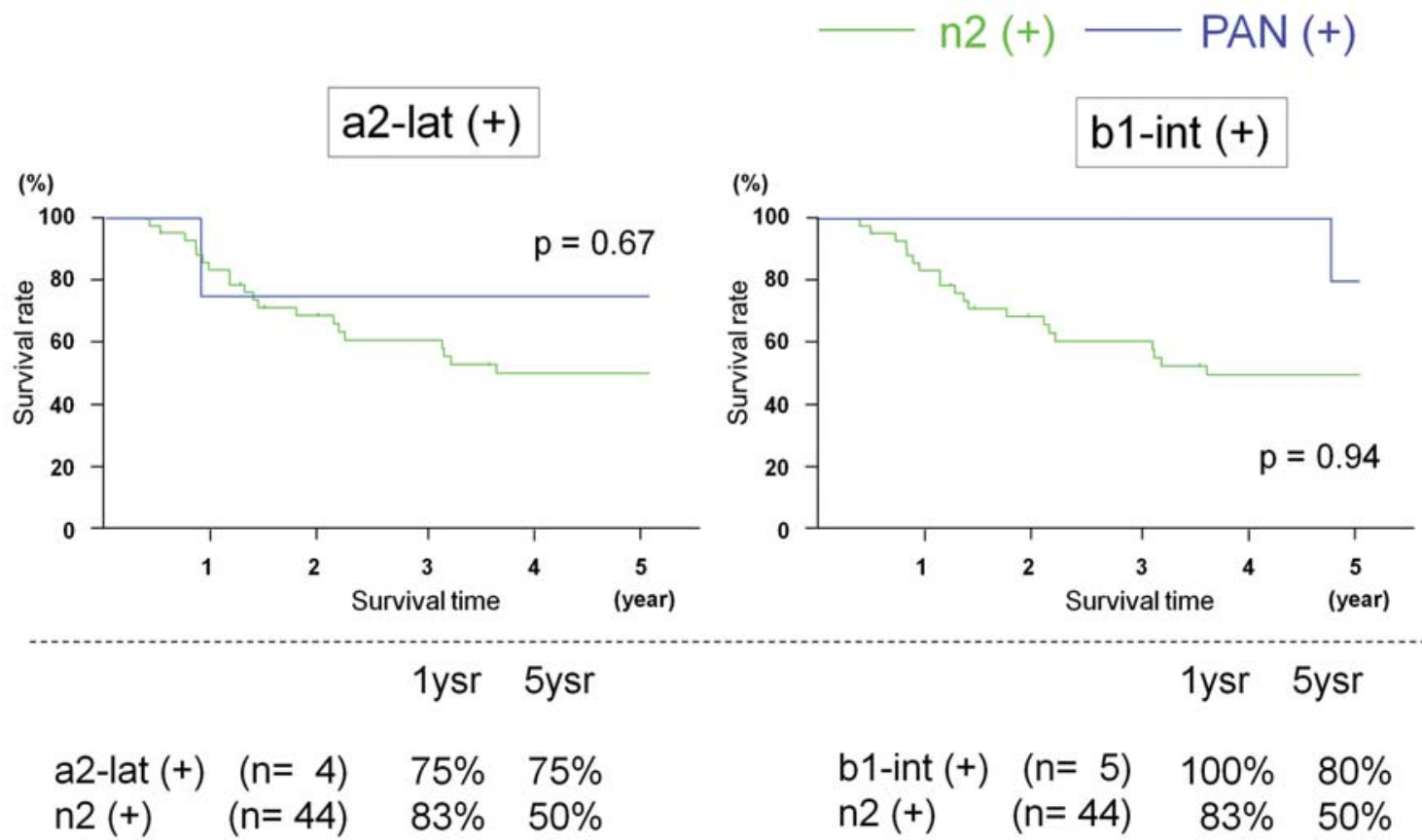

Figure 5. Survival curves of the $\mathrm{n} 2(+)$ group and the a2-lat $(+)$ or the b1-int $(+)$ group of the middle-third gastric cancer. There was no difference in the median survival times between the $\mathrm{n} 2(+)$ group and the a2-lat $(+)$ or the b1-int $(+)$ group in the middle-third stomach.

disclose difficulty in selection of real candidates for PAN dissection, that is, patients with PAN metastasis.

There are many studies describing risk factors preoperatively predicting PAN metastases. Macroscopic N stage
(N2 to N4) and tumor size ( $\geq 5 \mathrm{~cm}$ in diameter) were associated with PAN metastasis in the JCOG study 9501 (12). But only $20 \%$ of patients were N2 to N4 and $13 \%$ having tumor with $>5 \mathrm{~cm}$ had actual PAN metastasis in this study. Thus, these 


\section{Lower-third (the region L)}
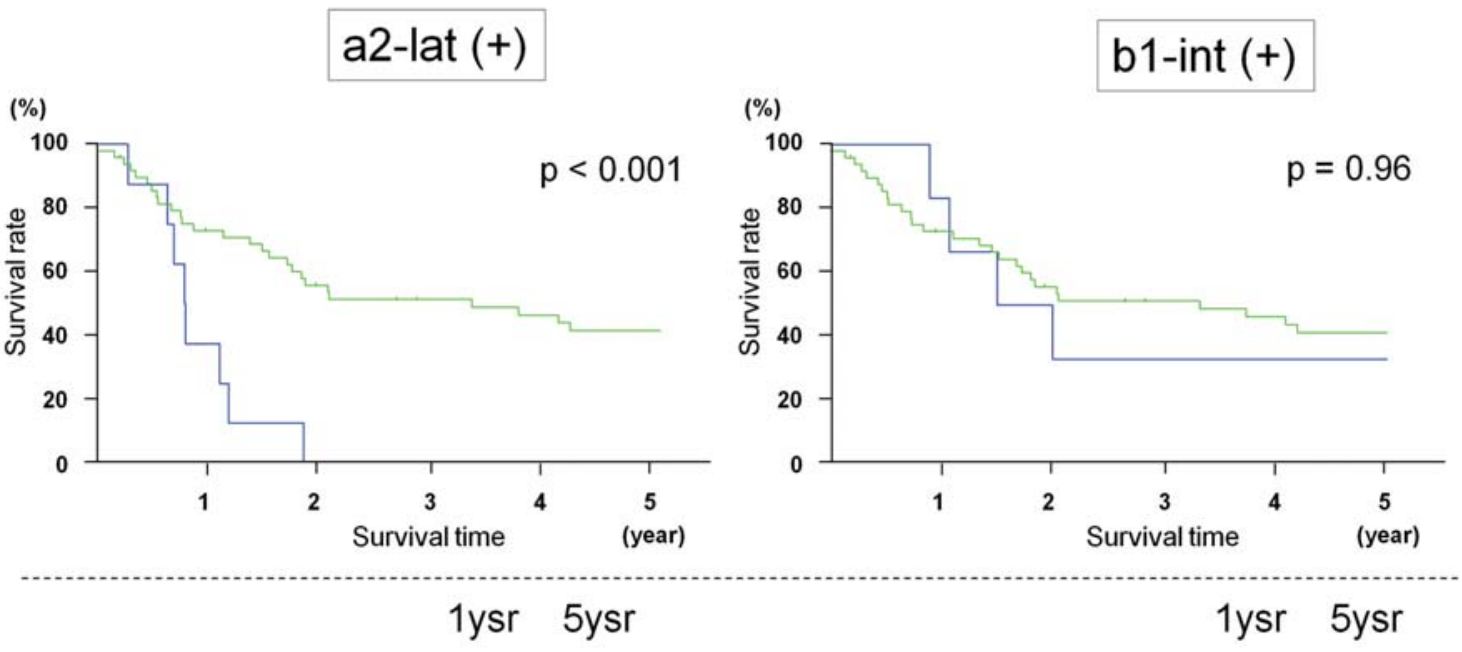

$\begin{array}{llrr}\text { a2-lat }(+) & (n=8) & 38 \% & 0 \% \\ \text { n2 }(+) & (n=50) & 73 \% & 42 \%\end{array}$

$\begin{array}{lrrr}\text { b1-int }(+) & (\mathrm{n}=6) & 83 \% & 33 \% \\ \mathrm{n} 2(+) & (\mathrm{n}=50) & 73 \% & 42 \%\end{array}$

Figure 6. Survival curves of the n2 (+) group and the a2-lat $(+)$ or the b1-int $(+)$ group of the lower-third gastric cancer. There was no difference in the median survival times between the $\mathrm{n} 2(+)$ group and the b1-int $(+)$ group, but the survival time of the $\mathrm{n} 2(+)$ group was significantly longer than that of the a2-lat $(+)$ group.

predictive factors may be necessary conditions, but not sufficient ones. Other risk factors included depth of tumor invasion (13), total number of metastatic lymph nodes (14) and lymphatic metastases to the stations \#7 and \#8 (15). It is difficult, however, intraoperatively to prove whether patients fit these risk factors. On the other hand, several factors indicating good prognosis of the patients with PAN metastasis, including number of metastatic PANs $(<3$ or 4$)$ $(6,16)$ and total number of metastatic lymph nodes $(<11)(16)$. But, again, it is hard to obtain such information before or during operation. These facts explain the difficulty in the patient selection for PAN dissection. We have performed PAN dissection to patients of gastric cancer without severe serosal invasion, but with lymph node metastasis to No. 3, 7, or 9 and showing $\mathrm{H} 0, \mathrm{P} 0, \mathrm{M} 0$ and $\mathrm{CyO}$ (17).

Lymphatic flow from the stomach drains into the perigastric nodes, next the node around the celiac axis or its main branches and finally into the PANs before joining the cisterna chyli as a systemic circulation. The main lymphatic route from the stomach to the PAN differs between the primary tumor locations. Research on lymphatic routes from perigastric nodes to PANs have been carried out using various tracers such as dyes, charcoals and radioisotopes. Yonemura (5) classified these lymphatic flows into 4 routes: i) Left subdiaphragmatic pedicle; ii) Celiac pedicle; iii) Superior mesenteric pedicle; and iv) Retropancreatic pedicle. The left subdiaphragmatic pedicle is characteristic of upperthird gastric cancer, especially, cardia cancer. This lymphatic flow reaches PANs next to the left side of aorta, that is, a2-lat. The lymphatic flow of the upper-third of the stomach drains into a2-lat not only along this left subphrenic artery, but also along the left gastric artery and the celiac axis. In this sense, a2-lat is likely to be important nodes for the upper-third gastric cancer. On the other hand, both the superior mesenteric and retropancreatic pedicles are characteristic of the lower-third gastric cancer. These lymphatic channels finally connect to the PANs next to the right side of aorta including a2-int and b1-int. The celiac pedicle is supposed to be a route common to whole area of the stomach. Metastasis to the PANs is strongly related with these lymphatic routes to the PANs. It is reported that PAN metastasis in the upper-third gastric cancer frequently occurs on the left side of aorta, while PAN metastasis in the lower-third gastric cancer tends to occur on the right side of aorta $(14,18)$.

Nishi reported that the prognosis of a2-lat metastasis in the upper-third gastric cancer is very close to the one of $\mathrm{n} 2$ metastasis (18). Sasako et al (19) summarized a nationwide questionnaire asking location tendency in subgroups of PANs in the patients who received para-aortic lymphadenectomy and survived longer than 5 years after surgery. This study indicated that the most and the second most of the survivors in the 61 patients had metastases in a2-lat $(n=31)$ and b1-int $(n=11)$, respectively. Most of the survivors according to the sub-groups by the tumor location were registered at the a2-lat in the upper third and the middle third cancer $(n=16$ and $n=7$, respectively) and b1-int in the lower third cancer $(n=7)$. These data strongly support our conception of selective lymphadenectomy of subgroups of PANs according to the tumor location.

Sano et al (4) reported that there was no significant difference in postoperative morbidity or mortality between D2 and D3, but in the D3 group volume of blood loss was high 
and the hospitalization was longer compared to the D2 group in the JCOG study 9501. Another randomized study conducted by Yonemura et al (20) demonstrated that both morbidity and mortality in the D3 group were significantly higher than those in the D2 group. If an advantage in elongation of survival would be achieved by the D3 operation, a little higher post-operative morbidity may be allowable, but two major randomized studies have failed in demonstrating survival benefits by PAN dissection $(8,21)$. In this situation, whole lymphadenectomy of the PANs should be avoided for all the patients with advanced gastric cancer. It is important to balance survival advantage with postoperative morbidities in the treatment of these patients.

\section{Acknowledgements}

The authors are grateful to Koichi Miwa, ex-professor of Kanazawa University and Yutaka Yonemura for their performance of surgery.

\section{References}

1. Bonenkamp JJ, Hermans J, Sasako M, van de Velde CJ, Welvaart K, Songun I, Meyer S, Plukker JT, Van Elk P, Obertop H, Gouma DJ, van Lanschot JJ, Taat CW, de Graaf PW, von Meyenfeldt MF, Tilanus H and Dutch Gastric Cancer Group: Extended lymph-node dissection for gastric cancer. N Engl J Med 340: 908-914, 1999 .

2. Cuschieri A, Weeden S, Fielding J, Bancewicz J, Craven J, Joypaul V, Sydes M and Fayers P: Patient survival after D1 and D2 resections for gastric cancer: long-term results of the MRC randomized surgical trial. Br J Cancer 79: 1522-1530, 1999.

3. Wu CW, Hsiung CA, Lo SS, Hsieh MC, Chen JH, Li AF, Lui WY and Whang-Peng J: Nodal dissection for patients with gastric cancer: a randomized controlled trial. Lancet Oncol 7: 309-315, 2006.

4. Sano T, Sasako M, Yamamoto S, Nashimoto A, Kurita A, Hiratsuka M, Tsujinaka T, Kinoshita T, Arai K, Yamamura Y and Okajima K: Gastric cancer surgery: morbidity and mortality results from a prospective randomized controlled trial comparing D2 and extended para-aortic lymphadenectomyJapan Clinical Oncology Group study 9501. J Clin Oncol 22: 2767-2773, 2004.

5. Yonemura Y: Lymphatic systems of the stomach and the patterns of lymph node metastasis. In: Contemporary Approaches Toward Cure of Gastric Cancer. Yonemura Y (ed). Maeda Shoten, Kanazawa, pp3-18, 1996.

6. Isozaki H, Okajima K, Fujii K, Nomura E, Izumi N, Mabuchi H, Nakamura $M$ and Hara $\mathrm{H}$ : Effectiveness of paraaortic lymph node dissection for advanced gastric cancer. Hepatogastroenterology 46: 549-554, 1999.

7. Kunisaki C, Shimada H, Yamaoka H, Wakasugi J, Takahashi M, Akiyama H, Nomura M and Moriwaki Y: Significance of para-aortic lymph node dissection in advanced gastric cancer. Hepatogastroenterology 46: 2635-2642, 1999.
8. Sasako M, Sano T, Yamamoto S, Kurokawa Y, Nashimoto A, Kurita A, Hiratsuka M, Tsujinaka T, Kinoshita T, Arai K, Yamamura Y, Okajima K and Japan Clinical Oncology Group: D2 lymphadenectomy alone or with para-aortic nodal dissection for gastric cancer. N Engl J Med 359: 453-462, 2008.

9. Sobin LH and Wittekind C: TNM Classification of Malignant Tumours. 6th edition. Wiley-Liss, New York, 2002.

10. Japanese Research Society for Gastric Cancer: Japanese Classification of Gastric Carcinoma. 2nd English edition. Kanehara, Tokyo, 1998.

11. Japanese Research Society for Gastric Cancer: Japanese Classification of Gastric Carcinoma. 1st English edition. Kanehara, Tokyo, 1995.

12. Nomura E, Sasako M, Yamamoto S, Sano T, Tsujinaka T, Kinoshita T, Furukawa H, Shimizu T, Hiratsuka M, Kobayashi O, Kurokawa Y, Tanigawa N and Gastric Cancer Surgical Study Group of the Japan Clinical Oncology Group: Risk factors for para-aortic lymph node metastasis of gastric cancer from a randomized controlled trial of JCOG9501. Jpn J Clin Oncol 37: 429-433, 2007.

13. Kosaka T, Usami K, Ueshige N, Hasegawa T, Yoshitani S, Sugaya J, Nakano Y and Takashima S: Paraaortic lymph node dissection for gastric cancer in 244 consecutive cases. Hepatogastroenterology 53: 629-633, 2006.

14. Baba M, Hokita S, Natsugoe S, Miyazono T, Shimada M, Nakano S, Takao S and Aikou T: Paraaortic lymphadenectomy in patients with advanced carcinoma of the upper-third of the stomach. Hepatogastroenterology 47: 893-896, 2000.

15. Yonemura Y, Wu CC, Fukushima N, Honda I, Bandou E, Kawamura T, Kamata S, Yamamoto H, Kim BS, Matsuki N, Sawa T, Noh SH and East Asia Surgical Oncology Group: Metastasis in para-aortic lymph nodes in patients with advanced gastric cancer, treated with extended lymphadenectomy. Hepatogastroenterology 54: 634-638, 2007.

16. Kunisaki C, Shimada H, Yamaoka H, Takahashi M, Ookubo K, Akiyama $\mathrm{H}$, Nomura $\mathrm{M}$ and Moriwaki $\mathrm{Y}$ : Indications for paraaortic lymph node dissection in gastric cancer patients with paraaortic lymph node involvement. Hepatogastroenterology 47: 586-589, 2000

17. Miwa K and Fujimura T: Indications of paraaortic lymph node dissection in gastric cancer from the viewpoint of long survivors. Geka Chiryo (in Japanese) 84: 562-567, 2001.

18. Nishi M: Para-aortic lymph node metastases in gastric cancer. In: Gastric Cancer Surgery Based on Clinicopathological Features. Nakayama Institute of Cancer Research, Tokyo, pp3944, 1995.

19. Sasako M, Sano T, Katai H and Maruyama K: Problems of the lymph node grouping in the Japanese classification of gastric carcinoma. Shujutsu (in Japanese) 52: 737-743, 1998.

20. Yonemura Y, Wu CC, Fukushima N, Honda I, Bandou E, Kawamura T, Kamata S, Yamamoto H, Kim BS, Matsuki N, Sawa T, Noh SH and East Asia Surgical Oncology Group: Operative morbidity and mortality after D2 and D4 extended dissection for advanced gastric cancer: a prospective randomized trial conducted by Asian surgeons. Hepatogastroenterology 53: 389-394, 2006.

21. Yonemura Y, Wu CC, Fukushima N, Honda I, Bandou E, Kawamura T, Kamata S, Kim BS, Matsuki N, Sawa T, Noh SH and East Asia Surgical Oncology Group: Randomized clinical trail of D2 and extended paraaortic lymphadenectomy in patients with gastric cancer. Int J Clin Oncol 13: 132-137, 2008. 